\title{
A novel wheat variety with elevated content of amylose increases resistant starch formation and may beneficially influence glycaemia in healthy subjects
}

\author{
Elinor Hallström', Francesco Sestili', Domenico Lafiandra², \\ Inger Björck' and Elin Östman'*
}

'Division of Applied Nutrition and Food Chemistry, Department of Food Technology, Engineering and Nutrition, Lund University, Lund, Sweden; 'Department of Agrobiology and Agrochemistry, University of Tuscia, Viterbo, Italy

Abstract

Background: Previous studies indicate that elevated amylose content in products from rice, corn, and barley induce lower postprandial glycaemic responses and higher levels of resistant starch (RS). Consumption of slowly digestible carbohydrates and RS has been associated with health benefits such as decreased risk of diabetes and cardiovascular disease.

Objective: To evaluate the postprandial glucose and insulin responses in vivo to bread products based on a novel wheat genotype with elevated amylose content (38\%).

Design: Bread was baked from a unique wheat genotype with elevated amylose content, using baking conditions known to promote amylose retrogradation. Included test products were bread based on whole grain wheat with elevated amylose content (EAW), EAW with added lactic acid (EAW-la), and ordinary whole grain wheat bread (WGW). All test breads were baked at pumpernickel conditions ( 20 hours, $\left.120^{\circ} \mathrm{C}\right)$. A conventionally baked white wheat bread (REF) was used as reference. Resistant starch (RS) content was measured in vitro and postprandial glucose and insulin responses were tested in 14 healthy subjects.

Results: The results showed a significantly higher RS content (on total starch basis) in breads based on EAW than in WGW $(p<0.001)$. Lactic acid further increased RS $(p<0.001)$ compared with both WGW and EAW. Breads baked with EAW induced lower postprandial glucose response than REF during the first 120 min $(p<$ $0.05)$, but there were no significant differences in insulin responses. Increased RS content per test portion was correlated to a reduced glycaemic index (GI) $(r=-0.571, p<0.001)$.

Conclusions: This study indicates that wheat with elevated amylose content may be preferable to other wheat genotypes considering RS formation. Further research is needed to test the hypothesis that bread with elevated amylose content can improve postprandial glycaemic response.

Keywords: resistant starch; retrogradation; amyloselamylopectin ratio; glycaemic response; insulinaemic response; lactic acid; anti-diabetic food; bread; wheat; whole grain

Received: I6 March 20II; Revised: 29 June 20I I; Accepted: I2 July 20II; Published: 22 August 20I I

\section{A} s reported by Stone and Morell (1), starch structure and technological functionality can impact human gastrointestinal physiology and it is likely that these characteristics can be exploited in an attempt to counteract the increasing incidence of lifestyle diseases. Previous studies on products based on genotypes with elevated amylose contents from barley, corn, and rice indicate that this type of starch induces lower postprandial glycaemic responses and also contains higher levels of resistant starch (RS) compared with starch of normal amylose contents (2-8). Possible health benefits from consumption of slowly digested carbohydrates and RS include improved metabolic control and decreased risk of cardiovascular disease, obesity, and type 1 and 2 diabetes (9). RS has also been shown to have positive effects on colonic health by increasing faecal bulk and by generating short chain fatty acids (SCFAs). The latter reduce $\mathrm{pH}$ in the colon and provide energy to the epithelial cells (10). Intake of RS has in particular been associated with colonic production of butyric acid, 
which is the main energy source for colonocytes and may, therefore, have a protective role in inflammatory bowel diseases and colon cancer (10-12).

In wheat, starch constitutes $65 \%-75 \%$ of the grain dry weight, with amylose contributing with $20 \%-30 \%$ of the total starch and amylopectin with the remaining $70 \%-80 \%$ (13). Elucidation of the role of enzymes involved in the starch biosynthetic pathway along with the isolation of genetic variants for these enzymes has provided an approach for the manipulation of the amylose/amylopectin ratio in bread and durum wheat, respectively $(1,14)$. Different approaches for increasing the amount of amylose content in wheat are available (15) and studies on the functional characteristics and possible uses of wheat lines with elevated amylose contents are currently investigated $(14,16)$. In particular, in the mid-1990s, Yamamori and Endo (17) analysed several bread wheat cultivars and identified natural mutants lacking one of the three possible isoforms of the starch synthase IIa protein. A combination of the different variants, in a line simultaneously lacking the three protein isoforms (null line), was achieved crossing the three different wheat varieties. The complete null line showed a significant increase in amylose content $(30.8 \%-$ $37.4 \%$ depending on the method used) compared to normal wheat varieties (18). In this study the metabolic response of a novel wheat genotype, similar to the one obtained by Yamamori and Endo (17), with elevated amylose content (38\%), was studied.

The suggested mechanism for how an increased amylose/amylopectin ratio affects glycaemic response is that the linear amylose chains form a compact structure that limits enzyme accessibility and rate of amylolysis ( 3,5 , 19). Amylopectin, on the other hand, with its branched structure and several terminal endings is less ordered than amylose and, therefore, more easily digested $(10,19)$.

In vitro studies on cereal genotypes with elevated amylose contents show a relationship between amylose content and amount of RS formed during processing $(3,7$, $10,20)$. The $\mathrm{RS}$ is defined as the fraction of starch that is not hydrolysed in the small intestine (10). Heating of starch in the presence of water will lead to gelatinisation that makes starch more easily digested. However, after cooling amylose tends to recrystallise and form retrograded amylose, which is unavailable for enzymatic hydrolysis (10). The principal form of RS in products based on starch with elevated amylose content is retrograded amylose $(16,21)$.

Intake of food products rich in RS has previously been associated with low postprandial glucose and insulin responses $(5,7)$. One suggested mechanism for this is that the process of retrogradation can transform gelatinised starch from an amorphous to a more crystalline state, which will increase enzyme resistance and reduce the rate of digestion (22). In agreement with this, previous studies on high-amylose corn in arepa products indicated that the
RS content influences not only the amount of unavailable starch but also the digestibility of available starch (7).

In the present study test breads were prepared at pumpernickel conditions ( 20 hours, $120^{\circ} \mathrm{C}$ ). A lower temperature allows endogenous enzymes to remain active during baking, which may enhance, for example, debranching of amylopectin (2). This will result in more linear glucose chains that can retrograde more easily and thereby contribute to RS formation. Previous studies thus indicate that baking at long time/low temperature prevents disruption of the physical and botanical structure of ingredients and results in a prolonged digestion $(2,23$, 24). In addition, lactic acid has been suggested to promote linearisation of amylopectin $(24,25)$ and limit enzyme accessibility in bread (26)

The aim of the present study was to evaluate the postprandial glucose and insulin responses in vivo to pumpernickel baked bread products based on a novel wheat genotype with elevated amylose content (38\%). The extent of starch retrogradation was estimated from the content of RS in the bread products and lactic acid was added to investigate if it could promote further RS formation. Commercial whole grain wheat was used to make possible a comparison between elevated and normal amylose levels, and all test products were served as equivalent loads of available carbohydrates.

\section{Material and methods}

\section{Study design}

Healthy subjects (seven females and seven males) were recruited to a randomised cross-over trial. Subjects were aged 20-35 years and had normal body mass indices (mean $\pm \mathrm{SD} ; 22.2 \pm 1.91$ ). In an attempt to standardise some of the factors that may influence glucose and insulin metabolism the subjects were asked not to use tobacco, antibiotics, or probiotics during and 2 weeks before the test period.

The day before each test day, subjects were asked to avoid strenuous exercise, drinking alcohol, or eat meals rich in fibre (i.e. legumes and wholegrain bread). Subjects were instructed to eat a low fibre dinner at 1800 hours and standardise their intakes by eating the same type of meal at the same time before all four test days. Subjects were also asked to eat a standardised evening meal consisting of 1-3 slices of white wheat bread (Dollar Storfranska, Lockarp, Sweden) with optional spread and drink between 2100 hours and 2200 hours. The same number of bread slices accompanied with the same spread and drink should be eaten before all four test days. The participants were not allowed to drink or eat anything after the standardised evening meal until the test meal in the morning. To optimise the standardisation regarding food intake, reminders were sent out to the participants and a follow-up was done at each test day, when the 
participants filled in a questionnaire describing their behaviour the day before.

The test meals consisted of $50 \mathrm{~g}$ available starch (calculated as total starch - RS) from each of the test breads and $250 \mathrm{ml}$ water. Subjects were served the test meals in random order at 0800 hours after the night long fast, on four different occasions between February and March 2010. Test meals were finished in 12-15 mins and postprandial glucose and insulin responses were measured by finger prick capillary blood sampling prior to intake (0) and at $15,30,45,60,90,120$, and $180 \mathrm{~min}$, respectively, after each experimental breakfast. After finishing the meal, subjects were not allowed to eat or drink until the last blood sample was taken. Glucose values were measured as $\mathrm{mmol} / \mathrm{l}$ plasma directly after sampling using a glucose oxidase method (HemoCue $201^{+}$, HemoCue AB, Angelholm, Sweden). Blood samples for insulin analysis were centrifuged and serum was separated and frozen. The insulin content was determined as nmol/l serum with an enzyme immunoassay kit (Mercodia AB, Uppsala, Sweden).

The Ethics Committee of the Faculty of Medicine at Lund University approved the study.

\section{Raw materials}

Whole grain flour from a novel wheat genotype with elevated amylose content (38\%) was provided by the University of Tuscia, Italy. The single SSIIa mutants identified by Yamamori and Endo (17) have been crossed and backcrossed three times with a breeding bread wheat line (N11). Subsequently, the three single null lines have been crossed between them to isolate a triple mutant line. Plants of the genotype with elevated amylose content were field grown under common cultural practices in the experimental station of the University of Tuscia (Viterbo, Central Italy). The whole grain wheat flour was milled and separated in three fractions (bran, middlings, and flour) before shipment to Lund University. The three fractions were mixed back into a whole grain flour with the proportions 1:1.06:3.55 (middlings:flour:bran) before used for bread baking and subsequent nutritional evaluation.

Besides the wheat flour described above, white wheat flour (Axa Kungsörnen, Järna, Sweden), whole grain wheat flour (Axa Kornkammaren, Järna, Sweden), gluten from wheat (SIGMA-ALDRICH, St Louis, MO, 80\% protein), salt with iodine (AB Hanson \& Möhrling, Halmstad, Sweden), yeast (Jästbolaget AB, Sollentuna, Sweden), and lactic acid (SIGMA-ALDRICH, ST Louis, MO, $88 \%-92 \%$ ), were used to prepare the bread products. All ingredients were food grade.

\section{Recipes and baking conditions}

Four different bread products were prepared, three pumpernickel baked breads and one conventionally baked reference bread from white wheat flour (REF). Two of the pumpernickel breads were based on whole grain wheat with elevated amylose content, one of them contained lactic acid (EAW-la) and one did not (EAW). The third pumpernickel bread was based on common whole grain wheat with normal amylose content $(25 \%-$ $30 \%$ ) (WGW).

The recipes were the following:

EAW: $46 \mathrm{~g}$ fresh yeast, $250 \mathrm{~g}$ water, $225 \mathrm{~g}$ EAW flour, $84 \mathrm{~g}$ white wheat flour, $16.3 \mathrm{~g}$ gluten and $2.5 \mathrm{~g}$ salt

EAW-la: same recipe as EAW but with the addition of $4.3 \mathrm{~g}$ lactic acid.

WGW: same recipe as EAW but with $200 \mathrm{~g}$ water and $225 \mathrm{~g}$ whole grain wheat (WGW) flour instead of EAW flour

REF: 4.8 g dry yeast, $360 \mathrm{~g}$ water, $540 \mathrm{~g}$ white wheat flour, and $4.8 \mathrm{~g}$ salt

The EAW, EAW-la, and WGW were prepared in a KitchenAid $^{\mathrm{TM}}$ Mixer (KSM90, KitchenAid, St. Joseph, MI). Finger warm water and yeast were mixed at the lowest speed for $2 \mathrm{~min}$, thereafter all remaining ingredients were added and mixed at medium speed for an additional $12 \mathrm{~min}$. The dough was proofed in a baking form on top of the oven for one hour. The baking form was tightly covered with two layers of aluminium foil and then put in a laboratory scale oven (Electrolux, Martinez, GA) for 20 hours at $120^{\circ} \mathrm{C}$. Four loaves were baked at the time. The REF bread was baked in a home baking machine according to Liljeberg and Björck (27) and was used as a reference for calculation of glycaemic index (GI), glycaemic profile (GP), and insulinaemic index (II).

After baking, the bread was cooled for 2 hours (REF) and 5 hours (EAW, EAW-la, WGW), respectively, at room temperature. In order to decrease a possible variability in RS content between bakings, the crust was then removed and the breads were wrapped in aluminium foil and put in plastic bags. All breads were kept frozen until the day before the test day, when they were taken from the freezer and thawed at room temperature over night, still in the aluminium foil and plastic bag.

\section{Characterisation of starch in the bread products}

Amylose content of the EAW flour was determined according to the method described by Chrastil (28). Bread samples were milled to pass a $0.5 \mathrm{~mm}$ screen (Cyclotech, Tecator, Höganäs, Sweden) and solubilised with $\mathrm{KOH}$ to include retrograded starch in the total starch analysis (29). Total starch was then determined according to Holm et al. (30). The amount of RS was analysed in vitro on fresh bread samples according to Åkerberg et al. (31). 


\section{Calculations and statistical analysis}

Data are expressed as means \pm SEM. The incremental areas under the curve (iAUC) for glucose and insulin (0$180 \mathrm{~min}$ and $60-180 \mathrm{~min}$, respectively) were calculated using the trapezoid model and using the fasting levels as baseline (Graph Pad Prism, version 4.03, Graph Pad Software, San Diego, CA). Glycaemic index (GI) and insulinaemic index (II) were calculated from the $120 \mathrm{~min}$ incremental postprandial area for glucose and insulin, respectively, using REF as the reference (GI and II =100) (32). Glycaemic profile was calculated as the duration of the incremental postprandial blood glucose response divided with the blood glucose incremental peak (min/ $\mathrm{mM}$ ) (33). The incremental peak is defined as the highest value above fasting level, measured for an individual test subject. In cases where the glucose concentration remained above fasting for the entire $180 \mathrm{~min}$, the duration was set to $180 \mathrm{~min}$.

Time $\times$ treatment interactions were analysed using a mixed model (PROC MIXED in SAS release 8, SAS Institute Inc. Cary, NC) with repeated measures and an autoregressive covariance structure.

The data were analysed with a general linear model (ANOVA), including subject and meal in the model, followed by Tukey's multiple comparison test (MINITAB, release 14.13, Minitab Inc, State College, PA). In the cases of unevenly distributed residuals (tested with AndersonDarling and considered unevenly distributed when $p<0.05$ ), Box Cox transformation were performed on the data prior to the ANOVA. Correlation analysis was conducted to evaluate the relation among dependent measures with the use of Spearman's partial coefficients controlling for subjects (two tailed test) (SPSS software, version 16.0; SPSS Inc, Chicago, IL).

\section{Results}

Starch composition in bread products

The amylose content in EAW flour was 38\%. The characteristics of the test breads are shown in Table 1.
The amount of total starch was lower in EAW and EAWla compared to the breads baked from commercial whole grain and white wheat flours, respectively, resulting in larger test portions of the former. The RS content ranged from $2.9 \%$ (total starch basis) in REF to $19 \%$ in EAW-la. Both breads based on wheat with elevated amylose content contained significantly higher amounts of RS (total starch basis) than WGW and REF $(p<.001)$, respectively. The highest amount of RS was found in EAW-la, which had a significantly higher RS content than all other test products $(p<0.001)$.

\section{Postprandial glucose responses}

The mean incremental glucose response curves and corresponding data are shown in Fig. 1 and Table 2, respectively. No time $\times$ treatment interaction was found (0-180 $\mathrm{min}, p=0.11)$. Both EAW and EAW-la showed significantly lower incremental areas (iAUC 0-120 min) and GIs compared to REF ( $p<0.05)$. The corresponding values following WGW did not differ significantly from any of the other test breads. The incremental peaks for glucose after ingestion of EAW, EAW-la, and WGW, respectively, were significantly lower than after $\operatorname{REF}(p<$ $0.05)$, but no significant differences in peak values were found between the three whole grain wheat test breads. The glycaemic profile did not differ significantly between any of the test breads.

\section{Postprandial insulin responses}

The mean incremental serum insulin response curves and corresponding data are shown in Fig. 2 and Table 3, respectively. A significant treatment effect was found for insulin $(p<0.05)$ but no time $\times$ treatment interaction $(0$ $180 \mathrm{~min}, p>0.5$ ). No significant differences were found in incremental peak, incremental area, or II between the test breads. However, in the late postprandial phase (iAUC 60-180), EAW showed significantly higher iAUC than WGW and REF $(p<0.05)$.

Table 1. Composition of test breads

\begin{tabular}{lcccccc}
\hline Bread & FW (g/portion) & Total starch $(\%$ of FW) & RS $^{\mathrm{b}}$ (\% of FW) & Total starch (g/portion) & RS (g/portion) & RS (\% of total starch) \\
\hline REF & 127 & 40.7 & $1.2^{\mathrm{a}}$ & $5 \mathrm{I}$ & 1.5 & $2.9^{\mathrm{a}}$ \\
WGW & 165 & 33.1 & $2.9^{\mathrm{b}}$ & 55 & 4.8 & $8.7^{\mathrm{b}}$ \\
EAW & 248 & 23.2 & $3.1^{\mathrm{b}}$ & 58 & 7.7 & $13.0^{\mathrm{c}}$ \\
EAW-la & 259 & 23.7 & $4.4^{\mathrm{c}}$ & $6 \mathrm{I}$ & $11.0^{\mathrm{d}}$ & $19.0^{\mathrm{d}}$ \\
\hline
\end{tabular}

Abbreviations: FW - fresh weight, RS - resistant starch, REF - white wheat reference bread, WGW - whole grain wheat bread (normal amylose content), EAW - bread based on whole grain wheat with elevated amylose content, EAW-la - EAW with added lactic acid.

${ }^{a}$ Result presented as mean $(n=2)$.

${ }^{b}$ Result is presented as mean $(n=6)$.

Test portions contributed with $50 \mathrm{~g}$ available starch, calculated as total starch-RS. Values in each column not sharing the same letters were significantly different, $p<0.05$ (ANOVA, followed by Tukey's test). 


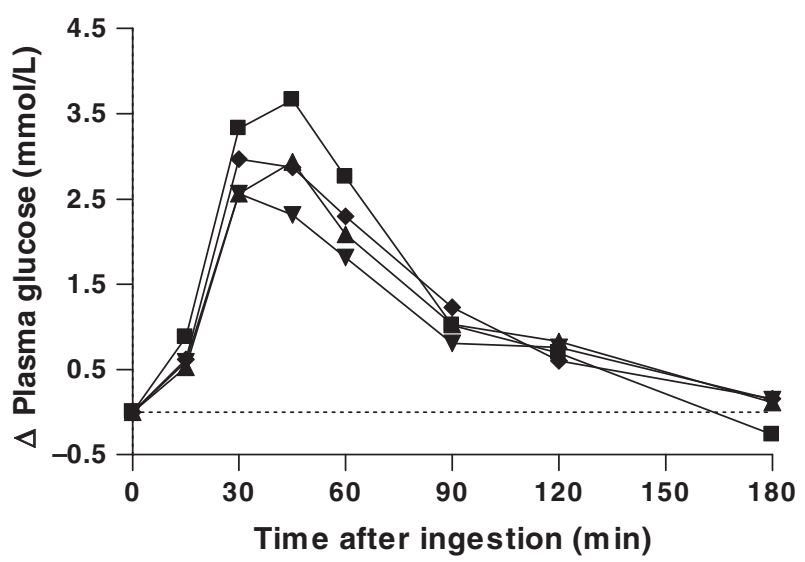

Fig. 1. Mean incremental change $(\Delta)$ in plasma glucose following intake of REF $(\boldsymbol{\bullet})$, WGW $(\bullet)$, EAW $(\boldsymbol{\Delta})$, and EAW-la $(\boldsymbol{\nabla}) . n=14$.

\section{Correlations}

Correlations $(n=14)$ between different parameters are shown in Table 4. The amount of RS in the test portions was negatively correlated to GI $(r=-0.571, p<0.001)$ and positively correlated to II $(r=0.286, p<0.05)$ and insulin incremental peak $(r=0.351, p<0.01)$. The GI was not correlated to II $(r=-0.086, p>0.05)$ but both GI $(r=-0.271, p<0.05)$ and II $(r=-0.329, p<0.05)$ were negatively correlated to GP.

\section{Discussion}

The present results show an increased RS formation in pumpernickel bread based on whole grain wheat with elevated amylose content (38\%) compared to a whole grain wheat genotype with normal amylose content $(20 \%-30 \%)$ baked at the same conditions. Increased RS formation in genotypes with elevated amylose content has previously been observed in bread based on corn, barley, and wheat (2, $5,34)$. Previous analysis of corn-based arepa bread showed a 4.5 times increase in RS content (starch basis) when the amylose level was changed from 25 to $70 \%$ (7). In the study by Åkerberg et al. (2) conventionally baked bread from waxy barley contained less than 1\% RS (starch basis), which increased to $4 \%$ when baking with a high amylose $(44 \%)$ barley genotype. In the latter study, baking at pumpernickel conditions further increased the RS content in the high amylose barley bread to $10 \%$ (starch basis). In the present study the RS content was around 1.5 times higher $(7.7 \mathrm{~g} /$ portion) in the EAW bread (38\% amylose) compared to bread baked with normal wheat genotype (4.8 g/portion). By adding lactic acid to the EAW bread, RS content was increased more than two times (11 g/portion) compared with the WGW bread. A complementary analysis performed in our laboratory (results not shown) showed that the RS content in conventionally baked EAW and EAW-la was 7\% (starch basis), respectively, compared to 13 and $19 \%$ when using pumpernickel baking. Consequently, when baked at conventional conditions, the EAW bread products contained twice as much RS as WGW (3.25\%, starch basis). This shows that conventional baking also yields significant amounts of RS in the case of EAW. Additionally, our results confirm that pumpernickel baking is an effective method in promoting $\mathrm{RS}$ formation.

In the present study, the EAW meal lowered the postprandial glucose responses significantly compared with REF. Similar studies have not previously been done on wheat but observations indicating that products with high amylose/amylopectin ratio lowers postprandial glycaemic responses have been reported for products made of barley, rice, and corn $(2,5,8)$. The correlation between increased RS content and reduced GI suggests that factors which promote the formation of enzyme RS also can influence the glycaemic response to the available carbohydrates. This is in line with previous findings showing that products rich in RS resulted in low glucose responses (7). It is important to emphasise that the enzyme resistant fraction of starch (RS) is not included in the test portion of $50 \mathrm{~g}$ available carbohydrates served in a GI study. This means that a lowered glucose response cannot be directly explained by a lowered available carbohydrate load. Instead our results seem to confirm the previous findings that formation of $\mathrm{RS}$ also can

Table 2. Blood glucose responses

\begin{tabular}{lcccc}
\hline Bread & iAUC 0-120 min $(\mathrm{min} \cdot \mathrm{mM})$ & Gl $(\%)$ & Glucose incremental peak $(\mathrm{mM})$ & GP $(\mathrm{min} / \mathrm{mM})$ \\
\hline REF & $224 \pm 27.3^{\mathrm{a}}$ & $100 \pm 0^{\mathrm{a}}$ & $4.0 \pm 0.3^{\mathrm{a}}$ & $38.7 \pm 3.7$ \\
WGW & $195 \pm 23.1^{\mathrm{a}, \mathrm{b}}$ & $89.2 \pm 6.8^{\mathrm{a}, \mathrm{b}}$ & $3.4 \pm 0.3^{\mathrm{b} *}$ & $3.0 \pm 0.3^{\mathrm{b} * *}$ \\
EAW & $185 \pm 29.7^{\mathrm{b} *}$ & $81.9 \pm 7.9^{\mathrm{b} *}$ & $3.0 \pm 0.2^{\mathrm{b} * *}$ & $49.9 \pm 7.9$ \\
EAW-la & $162 \pm 24.0^{\mathrm{b} * *}$ & $70.5 \pm 4.7^{\mathrm{b} * *}$ & $49.1 \pm 5.8$ \\
\hline
\end{tabular}

Abbreviations: REF - white wheat reference bread, WGW - whole grain wheat bread (normal amylose content), EAW - bread based on whole grain wheat with elevated amylose content, EAW-Ia - EAW with added lactic acid, GI - glycaemic index, GP - glycaemic profile, iAUC - incremental area under the curve.

Values are mean \pm SEM, $n=14$. Values in each column not sharing the same letters were significantly different, $p<0.05$ (ANOVA, followed by Tukey's test).

${ }^{*} p<0.05,{ }^{* *} p<0.01$. 


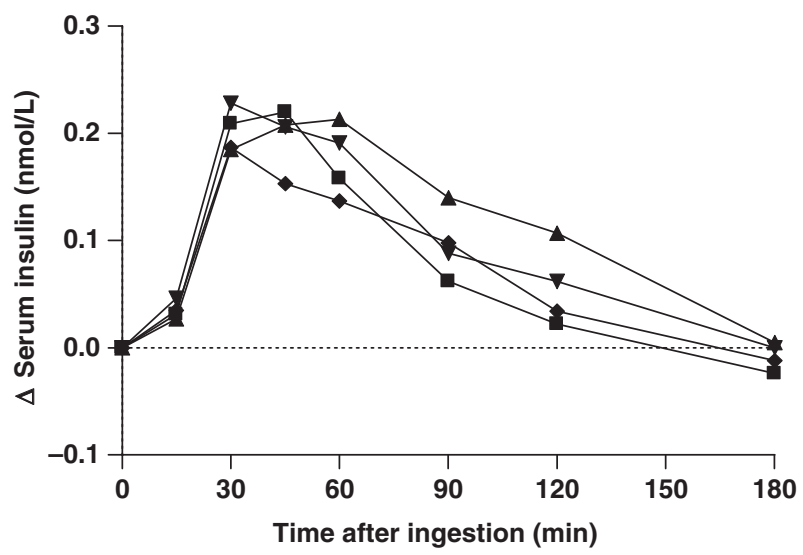

Fig. 2. Mean incremental change $(\Delta)$ in serum insulin following intake of REF $(\boldsymbol{\bullet})$, WGW $(\bullet)$, EAW $(\Delta)$, and EAW-la $(\boldsymbol{\nabla}) . n=14$.

influence the rate of digestion of the available starch fraction. However, this is the first time this has been shown for a wheat genotype with elevated amylose content. The compact structure of starch with elevated amylose content, which restricts swelling and gelatinisation, has been suggested to reduce rate of digestion (2, $35)$. Formation of complexes between amylose and surrounding lipids and proteins that resist enzymatic degradation may be additional factors contributing to a lower rate of starch digestion in products with elevated amylose contents $(36,37)$. Encapsulation of gelatinised starch between layers of RS has been proposed as another possible mechanism behind the increased content of a slowly digestible starch fraction (38), but more research is needed to investigate how starch is changing on a molecular level.

The EAW-la contained the highest amounts of RS and elicited the lowest glycaemic response of the test breads in the present study. Organic acids, such as acetic and propionic acid, are known to slow gastric emptying (19). In the case of lactic acid, previous in vitro studies (24) suggested that the rate of starch hydrolysis was reduced when lactic acid was added to starch/gluten mixtures prior to heat treatment. In the same study, an even lower rate of starch hydrolysis was noted in lactic acid bread when the gluten content was increased, although gluten alone showed no impact on starch hydrolysis. These observations indicate that lactic acid promotes interactions between starch and gluten and these interactions are suggested to create a barrier that can reduce the bioavailability of starch. Consequently, the prolonged time of starch gelatinisation due to a slow temperature increase during pumpernickel baking combined with the presence of lactic acid could further promote interactions between starch and gluten and, thereby, the formation of slowly digestible and enzyme-resistant starch. As mentioned previously in this report, the impact of lactic acid on RS formation could also be related to an increased linearisation of amylopectin due to enzymatic activity during pumpernickel baking (26).

The primary stimulus for insulin secretion is a change in blood glucose concentration. As the blood glucose levels decrease, also the stimuli of the beta cells will decline which normally results in a reduction of insulin secretion. In the present study, GI was not correlated to II and the EAW bread induced a larger late insulin secretion (iAUC 60-180 min) than WGW and REF bread, respectively, despite its lower GI. The delayed insulin peak $(t=60 \mathrm{~min})$ and the elevated late insulin response after ingestion of EAW, suggest that some insulin stimulating factor may have been absorbed in the late postprandial phase. The increased levels of insulin seen after ingestion of EAW could be a result of insulin stimulating peptides or amino acids being released during the pumpernickel baking. An insulin stimulating effect has previously been observed for nonglucose nutrients such as fatty acids and amino acids (39) and certain amino acids that are present in high amounts in whey can improve acute glucose regulation in both healthy subjects (40) as well as in patients with type 2 diabetes (41). It could not be excluded that the slow baking process may have promoted protein degradation by intrinsic enzymes. Another explanation for the increased

Table 3. Serum insulin response

\begin{tabular}{lcccc}
\hline Bread & Insulin incremental peak (nM) & iAUC 0-120 min (min.nM) & II (\%) & iAUC 60-180 min (min $\cdot n M)$ \\
\hline REF & $0.220 \pm 0.038$ & $12.9 \pm 1.96$ & $100 \pm 0$ & $5.2 \pm 1.01^{\mathrm{a} * * *}$ \\
WGW & $0.187 \pm 0.035$ & $12.2 \pm 1.82$ & $98 \pm 9.91$ & $6.2 \pm 1.19^{\mathrm{a} * *}$ \\
EAW & $0.213 \pm 0.037$ & $17.1 \pm 2.48$ & $155 \pm 29.36$ & $12.1 \pm 2.27^{\mathrm{b}}$ \\
EAW-la & $0.228 \pm 0.042$ & $15.2 \pm 1.98$ & $132 \pm 16.66$ & $8.1 \pm 1.27^{\mathrm{a}, \mathrm{b}}$ \\
\hline
\end{tabular}

Abbreviations: REF - white wheat reference bread, WGW - whole grain wheat bread (normal amylose content), EAW - bread based on whole grain wheat with elevated amylose content, EAW-Ia - EAW with added lactic acid, iAUC - incremental area under the curve.

Values are mean \pm SEM, $n=14$. Values in each column not sharing the same letters were significantly different, $p<0.05$ (ANOVA, followed by Tukey's test).

$*^{*} p<0.01$. 
Table 4. Correlations $(n=14)$ between RS, GI, iPeak, insulin iAUC 60-80 min, and GP

\begin{tabular}{|c|c|c|c|c|c|}
\hline & RS (g/portion) & $\mathrm{GI}(\%)$ & Insulin iPeak (nM) & Insulin iAUC 60-180 $\min (\min \cdot n M)$ & $\mathrm{GP}(\mathrm{min} / \mathrm{mM})$ \\
\hline II & $0.286 *$ & NS & $0.609 * * *$ & $0.657^{* * *}$ & $-0.329 *$ \\
\hline $\mathrm{GP}(\mathrm{min} / \mathrm{mM})$ & NS & $-0.27 I^{*}$ & NS & NS & \\
\hline Insulin iAUC $60-180 \min (\min \cdot n M)$ & $0.443 * *$ & NS & $0.373 * *$ & & \\
\hline Insulin iPeak (nM) & $0.35 I^{* *}$ & & & & \\
\hline GI (\%) & $-\left.0.57\right|^{* * * * ⿰ ㇇ ⿰ 亅 ⿱ 丿 丶 丶 ~}$ & & & & \\
\hline
\end{tabular}

Abbreviations: RS - resistant starch, GI - glycaemic index, GP - glycaemic profile, iAUC - incremental area under the curve, iPeak - insulin incremental peak.

${ }^{*} p<0.05,{ }^{* *} p<0.01,{ }^{* * *} p<0.00 \mathrm{I}, \mathrm{NS}=$ not significant (Spearman's partial coefficients controlling for subjects, two-tailed test).

insulin levels may be that the presence of a slowly digestible starch fraction may have resulted in a prolonged absorption of glucose and, thus, an increased insulin demand in the later postprandial phase. However, the late insulin increase does not seem proportionate to the glucose response when looking at mean values. In the present study an increased RS content was positively correlated to an increase in II. This is, however, not in line with previous studies where meals containing high-amylose starch have induced reductions in both postprandial glucose and insulin responses $(5,8,42)$. It should be noted though that the mentioned studies have investigated different forms of RS and that they may exert different effects in vivo. Two recent studies on rye products indicate that GP may be a better predictor of insulin response than GI $(33,43)$. Similar results were found in this study where the GP, but not GI, was correlated to II. The test breads did not differ in GP despite differences in glucose absorption. However all three pumpernickel baked breads showed a tendency of having higher values of GP than the reference bread. Taken together, the present study supports using the GP as a complement to GI in meal studies intended to describe postprandial glucose metabolism.

\section{Conclusion}

Using whole grain wheat with elevated amylose content significantly increased RS levels (starch basis) in both conventionally and pumpernickel baked whole grain wheat bread compared to normal wheat. The addition of lactic acid further promoted RS formation in pumpernickel baking of whole grain wheat with elevated amylose content. Besides the RS formation, pumpernickel baking also resulted in a slowly digestible starch fraction and lower postprandial glycaemia. No differences in insulin responses were found between the products. This study shows that wheat genotypes with elevated amylose content may be preferable to normal wheat genotypes considering RS content. Further research is needed to test the hypothesis that bread with elevated amylose content can improve glycaemic regulation.

\section{Acknowledgements}

We thank Dr M. Yamamori for supplying us the seeds of the single mutants of starch synthase IIa. We also thank Lisbeth Persson for her skilful analytical assistance.

\section{Conflict of interest and funding}

This study was financially supported by the European Commission in the Communities 6th Framework Programme, Project HEALTHGRAIN (FP6-514008). This publication reflects only author's views and the Community is not liable for any use that may be made of the information contained in this publication. Industrial partners were actively involved in the HEALTHGRAIN project. The authors declare no conflict of interest in relation to these partners.

\section{References}

1. Stone B, Morell M. Carbohydrate. In: Khan K, Shewry P, eds. Wheat: chemistry and technology. St Paul, MN: AACC International; 2009. p. 299-362.

2. Åkerberg A, Liljeberg H, Björck I. Effects of amylose/ amylopectin ratio and baking conditions on resistant starch formation and glycaemic indices. J Cereal Sci 1998; 28: 71-80.

3. Granfeldt Y, Liljeberg H, Drews A, Newman R, Björck I. Glucose and insulin responses to barley products - influence of food structure and amylose-amylopectin ratio. Am J Clin Nutr 1994; 59: 1075-82.

4. Behall KM, Hallfrisch J. Plasma glucose and insulin reduction after consumption of breads varying in amylose content. Eur J Clin Nutr 2002; 56: 913-20.

5. Granfeldt Y, Drews A, Björck I. Arepas made from high amylose corn flour produce favorable low glucose and insulin responses in healthy humans. J Nutr 1995; 125: 459-65.

6. Goddard MS, Young G, Marcus R. The effect of amylose content on insulin and glucose responses to ingested rice. Am $\mathrm{J}$ Clin Nutr 1984; 39: 388-92.

7. Granfeldt YE, Drews AW, Björck IME. Starch bioavailability in arepas made from ordinary or high amylose corn-concentration and gastrointestinal fate of resistant starch in rats. J Nutr 1993; 123: $1676-84$.

8. Brand Miller J, Pang E, Bramali L. Rice: a high or a low glycemic index food? Am J Clin Nutr 1992; 56: 1034-6.

9. Mann J, Cummings JH, Englyst HN, Key T, Liu S, Riccardi G, et al. FAO/WHO scientific update on carbohydrates in human nutrition: conclusions. Eur J Clin Nutr 2007; 61: S132-7. 
10. Sharma A, Yadav BS, Ritika BY. Resistant starch: physiological roles and food applications. Food Rev Int 2008; 24: 193-234.

11. Roediger WEW. Role of anaerobic-bacteria in the metabolic welfare of the colonic mucosa in man. Gut 1980; 21: 793-8.

12. Scheppach W, Sommer H, Kirchner T, Paganelli GM, Bartram P, Christl S, et al. Effect of butyrate enemas on the colonic mucosa in distal ulcerative-colitis. Gastroenterology 1992; 103: 51-6.

13. Cornell $\mathrm{H}$. The chemistry and biochemistry of wheat. In: Cauvain SP, ed. Bread making: improving quality. Sawston: Woodhead Publishing Limited; 2003. p. 31-70.

14. Lafiandra D, Sestili F, D'Ovidio R, Janni M, Botticella E, Ferrazzano G, et al. Approaches for modification of starch composition in durum wheat. Cereal Chem 2010; 87: 28-34.

15. Rahman S, Bird T, Regina A, Li Z, Ral JP, McMaugh S, et al. Resistant starch in cereals: exploiting genetic engineering and genetic variation. J Cereal Sci 2007; 46: 251-60.

16. Hung PV, Maeda T, Morita N. Waxy and high-amylose wheat starches and flours - characteristics, functionality and application. Trends Food Sci Technol 2006; 17: 448-56.

17. Yamamori M, Endo T. Variation of starch granule proteins and chromosome mapping of their coding genes in common wheat. Theor Appl Genetics 1996; 93: 275-81.

18. Yamamori M, Fujita S, Hayakawa K, Matsuki J, Yasui T. Genetic elimination of a starch granule protein, SGP-1, of wheat generates on altered starch with apparent high amylose. Theor Appl Genetics 2000; 101: 21-9.

19. Fardet A, Leenhardt F, Lioger D, Scalbert A, Rémésy C. Parameters controlling the glycaemic response to breads. Nutr Res Rev 2006; 19: 18-25.

20. Björck I, Eliasson A-C, Drews A, Gudmundsson M, Karlsson R. Some nutritional properties of starch and dietary fiber in barley genotypes containing different levels of amylose. Cereal Chem 1990; 67: 327-33.

21. Siljestrom M, Asp NG. Resistant starch formation during baking - effect of baking time and temperature and variations in the recipe. Zeitschrift Fur Lebensmittel-Untersuchung UndForschung 1985; 181: 4-8.

22. Chung H-J, Lim HS, Lim S-T. Effect of partial gelatinization and retrogradation on the enzymatic digestion of waxy rice starch. J Cereal Sci 2006; 43: 353-9.

23. Eerlingen RC, Crombez M, Delcour JA. Enzyme-resistant starch. 1. Quantitative and qualitative influence of incubationtime and temperature of autoclaved starch on resistant starch formation. Cereal Chem 1993; 70: 339-44.

24. Berry CS. Resistant starch-formation and measurement of starch that survives exhaustive digestion with amylolytic enzymes during the determination of dietary fiber. J Cereal Sci 1986; 4: 301-14.

25. Liljeberg H, Åkerberg A, Björck I. Resistant starch formation in bread as influenced by choice of ingredients or baking conditions. Food Chem 1996; 56: 389-94.

26. Östman EM, Nilsson M, Liljeberg Elmstahlt HGM, Molin G, Bjorck IME. On the effect of lactic acid on blood glucose and insulin responses to cereal products: mechanistic studies in healthy subjects and in vitro. J Cereal Sci 2002; 36: 339-46.

27. Liljeberg H, Björck I. Bioavailability of starch in bread products - postprandial glucose and insulin responses in healthy-subjects and in-vitro resistant starch content. Eur J Clin Nutr 1994; 48: $151-63$.

28. Chrastil J. Improved colorimetric determination of amylose in starches or flours. Carbohydr Res 1987; 159: 154-8.
29. Siljestrom M, Eliasson AC, Björck I. Characterization of resistant starch from autoclaved wheat-starch. Starch/Starke 1989; 41: 147-51.

30. Holm J, Björck I, Drews A, Asp N-G. A rapid method for the analysis of starch. Starch/Starke 1986; 38: 224-6.

31. Åkerberg AK, Liljeberg HG, Granfeldt YE, Drews AW, Björck IM. An in vitro method, based on chewing, to predict resistant starch content in foods allows parallel determination of potentially available starch and dietary fiber. J Nutr 1998; 128: 651-60.

32. Brouns F, Björck I, Frayn KN, Gibbs AL, Lang V, Slama G. Glycaemic index methodology. Nutr Res Rev 2005; 18: 145-71.

33. Rosén LA, Silva LO, Andersson UK, Holm C, Ostman EM, Björck IM. Endosperm and whole grain rye breads are characterized by low post-prandial insulin response and a beneficial blood glucose profile. Nutr J 2009; 8: 42.

34. Hung PV, Yamamori M, Morita N. Formation of enzymeresistant starch in bread as affected by high-amylose wheat flour substitutions. Cereal Chem 2005; 82: 690-4.

35. Panlasigui LN, Thompson LU, Juliano BO, Perez CM, Yiu SH, Greenberg GR. Rice varieties with similar amylose content differ in starch digestibility and glycemic response in humans. Am J Clin Nutr 1991; 54: 871-7.

36. Holm J, Björck I, Ostrowska S, Eliasson A-C, Asp N-G, Larsson K. Digestibility of amylose-lipid complexes in vitro and in vivo. Starch 1983; 35: 294-7.

37. Jenkins DJA, Wolever TMS, Thorne MJ, Rao AV, Thompson LU. Effect of starch-protein interaction in wheat on its digestibility and glycemic response. Canadian Institute of Food Science and Technology Journal-Journal De L Institut Canadien De Science Et Technologie Alimentaires 1987; 20: 320-320.

38. Zhang G, Ao Z, Hamaker BR. Slow digestion property of native cereal starches. Biomacromolecules 2006; 7: 3252-8.

39. Torres N, Noriega L, Tovar AR. Nutrient modulation of insulin secretion. Vitam Horm 2009; 80: 217-44.

40. Nilsson M, Holst JJ, Björck IM. Metabolic effects of amino acid mixtures and whey protein in healthy subjects: studies using glucose-equivalent drinks. Am J Clin Nutr 2007; 85: 996-1004.

41. Frid AH, Nilsson M, Holst JJ, Björck IM. Effect of whey on blood glucose and insulin responses to composite breakfast and lunch meals in type 2 diabetic subjects. Am J Clin Nutr 2005; 82: 69-75.

42. Behall KM, Scholfield DJ, Canary J. Effect of starch structure on glucose and insulin responses in adults. Am J Clin Nutr 1988; 47: 428-32.

43. Rosen LA, Östman EM, Björck IM. Effects of cereal breakfasts on postprandial glucose, appetite regulation and voluntary energy intake at a subsequent standardized lunch; focusing on rye products. Nutr J 2011; 10: 7.

\footnotetext{
*Elin Östman

Division of Applied Nutrition and Food Chemistry

Department of Food Technology, Engineering and Nutrition

Lund University

PO Box 124

SE-22I 00 Lund, Sweden

Tel: +46462228318

Fax: +46462224532

Email address: Elin.Ostman@appliednutrition.th.se
} 